\title{
ON EQUIVARIANT CHERN-WEIL FORMS AND DETERMINANT LINES
}

\author{
DANIEL S. FREED
}

\begin{abstract}
A strong from of invariance under a group $G$ is manifested in a family over the classifying space $B G$. We advocate a differential-geometric avatar of $B G$ when $G$ is a Lie group. Applied to $G$-equivariant connections on smooth principal or vector bundles, the equivariance $\rightarrow$ families principle converts the $G$-equivariant extensions of curvature and Chern-Weil forms to the standard nonequivariant versions. An application of this technique yields the moment map of the determinant line of a $G$-equivariant Dirac operator, which in turn sheds light on some anomaly formulas in quantum field theory.
\end{abstract}

Let $X$ be a smooth manifold, $H$ a Lie group, and $P \rightarrow X$ a smooth principal $H$-bundle with connection $\Theta$. Suppose that a Lie group $G$ acts on $P \rightarrow X$ preserving $\Theta$. If $G$ acts freely, so that there is a quotient principal $H$-bundle $P / G \rightarrow X / G$, then there is an obstruction to descending $\Theta$ to the quotient bundle: the moment map. This obstruction-defined for not-necessarily-free $G$ actions - is the key ingredient in a $G$-equivariant extension of the curvature of $\Theta$, so too in the $G$-equivariant extension of the Chern-Weil forms [BV], which live in $G$-equivariant de Rham theory.

Especially in topology and algebraic geometry a strong form of $G$-invariance is expressed by fibering over a classifying space $B G$. The particular form of the classifying space varies with context. Here we advocate in differential geometry for $B_{\nabla} G$, the classifying "generalized manifold" of $G$ connections introduced and studied in [FH]. As we review below, $B_{\nabla} G$ is a simplicial sheaf on the site of smooth manifolds, a generalized manifold in the same sense that a distribution is a generalized function. A $G$-manifold $X$ has a differential Borel quotient, which is a fiber bundle $\left(X_{G}\right)_{\nabla} \rightarrow B_{\nabla} G$ with fiber $X$, and we proved [FH, Theorem 7.28(ii)] that the de Rham complex of $\left(X_{G}\right)_{\nabla}$ is the Weil model for $G$-equivariant de Rham theory. Here, given a $G$-equivariant connection $\Theta$ on $P \rightarrow X$, the strong form of $G$-invariance is a connection $\Theta_{G}$ on the differential Borel quotient $\left(P_{G}\right)_{\nabla} \rightarrow\left(X_{G}\right)_{\nabla}$. Our main theorem identifies the curvature of $\Theta_{G}$ with the $G$-equivariant curvature of $\Theta$, and similarly the Chern-Weil forms of $\Theta_{G}$ with the $G$-equivariant Chern-Weil forms of $\Theta$. (A less rigid version of this construction was used in [BT] to prove that equivariant Chern-Weil forms represent equivariant characteristic classes.)

The differential Borel quotient converts equivariance into a fiber bundle. As an application of this technique we prove a theorem about $G$-equivariant families of Dirac operators. In [BF] we constructed a canonical connection on the determinant line bundle of a family and computed a formula for its curvature. Attached to a $G$-equivariant family of Dirac operators we obtain a $G$ equivariant determinant line bundle, and here we compute the corresponding moment map. The proof uses the equivariance $\rightarrow$ families construction to reduce the moment map computation to the known curvature formula. Applied to quantum field theory we find a direct geometric interpretation of standard "covariant" anomaly formulas in the physics literature (e.g., [BZ, ASZ, AgG, AgW]).

Date: June 6, 2016.

The work of D.S.F. is supported by the National Science Foundation under grant DMS-1207817. 


\section{Equivariant connections and $B_{\nabla} G$}

The main construction and computation lie squarely in differential geometry: no simplicial sheaves required. Let $\pi: P \rightarrow X$ be a fiber bundle and $W$ a horizontal distribution: $W \subset T P$ is a subbundle and the inclusion maps induce an isomorphism $W \oplus T(P / X) \cong T P$, where $T(P / X)=$ $\operatorname{ker} \pi_{*}$ is the subbundle of vertical vectors. If $\pi$ is a principal $H$-bundle for a Lie group $H$ and $W$ is $H$-invariant, then $W$ is a connection, but our construction is more general (and we need the general version in the next section). Let $G$ be a Lie group which acts on $\pi: P \rightarrow X$ and preserves the distribution $W$. Suppose $Q \rightarrow M$ is a principal $G$-bundle with connection $V \subset T Q$ a $G$-invariant horizontal distribution. Then the distribution $V \oplus W \subset T(Q \times P)$ is $G$-invariant, so descends to a horizontal distribution $W_{Q}$ on the quotient fiber bundle $\pi_{Q}$ in the iterated fibration

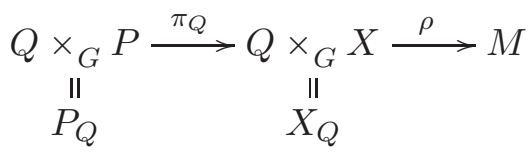

The restriction of $W_{Q}$ over a fiber of $\rho$ may be identified with $W$. The horizontal distribution $W_{Q}$ is functorial for maps of $G$-bundles with connection.

In case $\pi: P \rightarrow X$ is a principal $H$-bundle and $W$ a connection, we compute the curvature of $W_{Q}$. Let $\Theta \in \Omega_{P}^{1}(\mathfrak{h})$ be the connection form with kernel $W$. Assume for simplicity that $G$ is finite dimensional, and fix a basis $\left\{e_{a}\right\} \subset \mathfrak{g}$. Define the structure constants $f_{a b}^{c} \in \mathbb{R}$ by $\left[e_{a}, e_{b}\right]=f_{a b}^{c} e_{c}$. Each basis element $e_{a}$ induces a vector field on $P$, via the infinitesimal $G$-action, and so a contraction operator $\iota_{a}: \Omega_{P}^{\bullet} \rightarrow \Omega_{P}^{\bullet-1}$ of degree -1 on differential forms (with coefficients). Let $\phi=\phi^{a} e_{a} \in \Omega_{Q}^{1}(\mathfrak{g})$ be the connection form with kernel $V$. Let $\Omega=d \Theta+\frac{1}{2}[\Theta \wedge \Theta] \in \Omega_{P}^{2}(\mathfrak{h})$ and $\omega=d \phi+\frac{1}{2}[\phi \wedge \phi] \in \Omega_{Q}^{2}(\mathfrak{g})$ be the curvatures of $\Theta$ and $\phi$, respectively. Write $\omega=\omega^{a} e_{a}$. Finally, let $\Theta_{Q}, \Omega_{Q}$ denote the connection and curvature of the connection $W_{Q}$ on the principal $H$-bundle $\pi_{Q}$ in (1).

\section{Proposition 2.}

(i) $\Theta_{Q}=\Theta-\phi^{a} \cdot \iota_{a} \Theta$.

(ii) $\Omega_{Q}=\Omega-\phi^{a} \wedge \iota_{a} \Omega+\frac{1}{2} \phi^{a} \wedge \phi^{b} \cdot \iota_{b} \iota_{a} \Omega-\omega^{a} \cdot \iota_{a} \Theta$.

Proof. The form on the right hand side of (i) vanishes on $V \oplus W$ and is the identity on vertical vectors, since $\Theta$ is. For (ii) we compute using $\left(d \iota_{a}+\iota_{a} d\right) \Theta=0$ by $G$-invariance:

$$
\Omega_{Q}=\Omega-d\left(\phi^{a} \cdot \iota_{a} \Theta\right)-\left[\phi^{a} \cdot \iota_{a} \Theta \wedge \Theta\right]+\frac{1}{2}\left[\phi^{a} \cdot \iota_{a} \Theta \wedge \phi^{b} \cdot \iota_{b} \Theta\right]
$$

and

$$
-d\left(\phi^{a} \cdot \iota_{a} \Theta\right)=-\omega^{a} \wedge \iota_{a} \Theta+\frac{1}{2} f_{a b}^{c} \phi^{a} \wedge \phi^{b} \cdot \iota_{c} \Theta-\phi^{a} \wedge \iota_{a} \Omega+\phi^{a} \wedge\left[\iota_{a} \Theta, \Theta\right] .
$$

The $G$-invariance of $\Theta$ also implies

$$
\iota_{b} \iota_{a} \Omega=f_{a b}^{c} \iota_{c} \Theta+\left[\iota_{a} \Theta, \iota_{b} \Theta\right] .
$$

Combine (3), (4), and the product of (5) with $\frac{1}{2} \phi^{a} \wedge \phi^{b}$ to conclude. 
Let Man denote the category of smooth finite dimensional manifolds and smooth maps. A simplicial presheaf is a contravariant functor $\mathcal{F}$ from Man to the category of simplicial sets. It is a simplicial sheaf if it satisfies a covering condition; see $[\mathrm{FH}]$ and the references therein. An object $M \in$ Man is a "test manifold", and $\mathcal{F}(M)$ is the value of the sheaf $\mathcal{F}$ on that test manifold. The sheaves we consider have values in the category of groupoids: an object in $B_{\nabla} G(M)$ is a principal $G$-bundle $Q \rightarrow M$ with connection $\phi$ and a morphism is an isomorphism of principal $G$ bundles which preserves the connections. There is a universal $G$-bundle $E_{\nabla} G \rightarrow B_{\nabla} G$; an object in $E_{\nabla} G(M)$ is a principal $G$-bundle $Q \rightarrow M$ with connection and a section. A smooth finite dimensional manifold $X$ is a sheaf via the Yoneda embedding. Yoneda also implies that $\mathcal{F}(M)$ is the set of maps $M \rightarrow \mathcal{F}$ in the category of sheaves. If $X$ carries a left $G$-action, then we form the differential Borel construction

$$
\left(X_{G}\right)_{\nabla}:=E_{\nabla} G \times_{G} X \stackrel{\rho}{\longrightarrow} B_{\nabla} G
$$

a fiber bundle with fiber $X$. Therefore, given $\pi: P \rightarrow X$ which is $G$-equivariant, then (1) is the pullback of the iterated fiber bundle

$$
\left(P_{G}\right)_{\nabla} \stackrel{\pi_{G}}{\longrightarrow}\left(X_{G}\right)_{\nabla} \stackrel{\rho}{\longrightarrow} B_{\nabla} G
$$

via the map $M \rightarrow B_{\nabla} G$ represented by the principal $G$-bundle $Q \rightarrow M$ with connection $\phi$. If $W$ is a $G$-invariant horizontal distribution on $\pi$, then the construction at the beginning of this section gives a horizontal distribution $W_{G}$ on $\pi_{G}$. It encodes the "strong $G$-invariance" of $W$.

The de Rham complex of $E_{\nabla} G$ is the Weil algebra $\mathrm{Sym}^{\bullet} \mathfrak{g}^{*} \otimes \bigwedge^{\bullet} \mathfrak{g}^{*}$, which is the free differential graded algebra on $\mathfrak{g}^{*}=\bigwedge^{1} \mathfrak{g}^{*}$. Let $\left\{\theta^{a}\right\} \subset \bigwedge^{1} \mathfrak{g}^{*}$ be the dual basis to $\left\{e_{a}\right\} \subset \mathfrak{g}$; then $\left\{\chi^{a}=d \theta^{a}\right\} \subset$ $\mathrm{Sym}^{1} \mathfrak{g}^{*}$ is also a basis. Set $\nu^{a}=d \theta^{a}+\frac{1}{2} f_{b c}^{a} \theta^{b} \theta^{c}$. If $X$ is a smooth $G$-manifold, then the Weil model of equivariant de Rham theory is the basic subcomplex of $\operatorname{Sym}^{\bullet} \mathfrak{g}^{*} \otimes \bigwedge^{\bullet} \mathfrak{g}^{*} \otimes \Omega_{X}^{\bullet}$; see [MQ, §5], [GS]. Following H. Cartan [C1, C2], these references also construct a quasi-isomorphism with the Cartan model, the $G$-invariant subcomplex of Sym $\mathfrak{g}^{*} \otimes \Omega_{X}^{\bullet}$ with differential $d_{X}-\iota_{\xi}=d_{X}-\chi^{a} \iota_{a}$, where $\xi$ is the $G$-invariant $\mathfrak{g}^{*}$-valued vector field on $X$ which expresses the infinitesimal $G$-action. The quasi-isomorphism is the augmentation map of the exterior algebra: it sends $\theta^{a} \rightarrow 0, \nu^{a} \rightarrow \chi^{a}$ for all $a$. As quoted earlier from [FH], the de Rham complex of $\left(X_{G}\right)_{\nabla}$ is the Weil model of equivariant de Rham theory on $X$.

Theorem 8. Let $\pi: P \rightarrow X$ be a principal $H$-bundle with connection $\Theta$, and suppose $G$ acts on $P \rightarrow X$ preserving $\Theta$. Then the curvature of the induced connection on $\pi_{G}:\left(P_{G}\right)_{\nabla} \rightarrow\left(X_{G}\right)_{\nabla}$ is

$$
\Omega_{G}=\Omega-\chi^{a} \cdot \iota_{a} \Theta
$$

in the Cartan model.

Let $\mathfrak{g}_{P} \rightarrow X$ be the adjoint bundle of Lie algebras; then $\left(e_{a} \mapsto \iota_{a} \Theta\right) \in \operatorname{Hom}\left(\mathfrak{g}, \Omega_{X}^{0}\left(\mathfrak{g}_{P}\right)\right)$ is the moment map. $\Omega_{G}$ is the $G$-equivariant extension of the curvature defined in [BV, §2]; it is closed with respect to the covariant Cartan differential $d_{\Theta}-\iota_{\xi}$. 
Proof. The corresponding expression in the Weil model is

$$
\Omega-\theta^{a} \wedge \iota_{a} \Omega+\frac{1}{2} \theta^{a} \wedge \theta^{b} \cdot \iota_{b} \iota_{a} \Omega-\nu^{a} \cdot \iota_{a} \Theta .
$$

The map $M \rightarrow B_{\nabla} G$ given by $Q \rightarrow M$ with connection $\phi$ induces a pullback on de Rham complexes known as the Chern-Weil homomorphism [MQ]. It sends (10) to the curvature $\Omega_{Q}$ of the induced connection on $\pi_{G}$, by Proposition 2, which proves (9) since forms are determined by their pullbacks to test manifolds.

\section{Equivariant families of Dirac operators}

We translate the main construction (1) from principal bundles to vector bundles. Let $q: Y \rightarrow T$ be a principal $G$-bundle with connection $U \subset T Y$, and suppose $E \rightarrow Y$ is a vector bundle with a $G$-action and $G$-invariant covariant derivative $\nabla$. Define $q_{*} \nabla$ on $E / G \rightarrow T$ by

$$
\left(q_{*} \nabla\right)_{\xi} s=\nabla_{\tilde{\xi}}\left(q^{*} s\right)
$$

where $\tilde{\xi}$ is the horizontal lift of the tangent vector $\xi$ and $s$ is a section of $E / G \rightarrow T$. Given $\pi: E \rightarrow X$ with $G$-action and $G$-invariant $\nabla$, and a $G$-bundle $Q \rightarrow M$ with connection $V$, apply (11) to $Y=Q \times X, U=V \oplus T X$ to construct $\nabla_{Q}$ on $E_{Q} \rightarrow X_{Q}$ (i.e., $Q \times_{G} E \rightarrow Q \times_{G} X$ ).

Recall that if $E \rightarrow Y$ is a vector bundle with covariant derivative $\nabla$ and $E^{\prime} \underset{p}{\stackrel{i}{\rightleftarrows}} E$ is a complemented subbundle, then there is a compressed covariant derivative $\Pi_{E^{\prime}}^{E} \nabla=p \circ \nabla \circ i$ on $E^{\prime} \rightarrow Y$. The compression is transitive for iterated complemented subbundles $E^{\prime \prime} \rightleftarrows E^{\prime} \rightleftarrows E$.

Let $\pi: X \rightarrow S$ be a smooth fiber bundle. A relative Riemannian structure is a metric $g^{X / S}$ on $T(X / S) \rightarrow X$ together with a horizontal distribution $W \subset T X$. It determines a relative Levi-Civita covariant derivative $\nabla^{X / S}$ on the relative tangent bundle $T(X / S) \rightarrow X$ as follows. A Riemannian metric $g^{S}$ on $S$ induces a Riemannian metric $g^{X}$ on $X$ which makes $\pi$ a Riemannian submersion. Let $\nabla^{X}$ be the Levi-Civita covariant derivative on $X$. Then $\nabla^{X / S}=\Pi_{T(X / S)}^{T X} \nabla^{X}$ is independent of $g^{S}$. If the fibers of $\pi$ are closed manifolds, and if there is a relative spin structure, then there is an associated family of Dirac operators. Quillen [Q] constructed a metric on the determinant line bundle Det $\rightarrow S$; it carries a compatible covariant derivative whose curvature is the 2 -form component of the pushforward of the $\hat{A}$ polynomial applied to the relative curvature $[\mathrm{BF}]$ :

$$
\omega=2 \pi i\left[\int_{X / S} \hat{A}\left(\Omega^{X / S}\right)\right]_{(2)}
$$

There is an extension for families of generalized Dirac operators.

Now suppose a Lie group $G$ acts on $X \rightarrow S$ preserving all the data. (The "preservation" of the relative spin structure is additional data.) Then there is an induced $G$-action on Det $\rightarrow S$ which preserves the covariant derivative, and so an equivariant curvature

$$
\omega_{G}=\omega-\mu
$$

in the Cartan model, where $\mu \in \operatorname{Hom}\left(\mathfrak{g}, \Omega_{S}^{0}\right)$ is the moment map. 


\section{Theorem 14.}

$$
\omega_{G}=2 \pi i\left[\int_{X / S} \hat{A}\left(\Omega_{G}^{X / S}\right)\right]_{(2)}
$$

The equivariant curvature $\Omega_{G}^{X / S}$ is (9), and the integrand is the $G$-equivariant Chern-Weil form associated to the $\hat{A}$ polynomial. To compute the moment map it suffices to take $S=$ pt.

Lemma 16. Fix a Lie group $G$ and a principal $G$-bundle $Q \rightarrow M$ with connection.

(i) Let $X$ be a Riemannian manifold with Levi-Civita covariant derivative $\nabla$. Suppose $G$ acts on $X$ by isometries. Then $\nabla_{Q}$ is the relative Levi-Civita covariant derivative on $X_{Q} \rightarrow M$.

(ii) Let $\pi: X \rightarrow S$ be a fiber bundle with relative Riemannian structure $\left(g^{X / S}, W \subset T X\right)$; there is an induced relative Levi-Civita covariant derivative $\nabla^{X / S}$. If $G$ acts preserving all data, then $\nabla_{Q}^{X / S}$ is the relative Levi-Civita covariant derivative on $X_{Q} \rightarrow S_{Q}$.

Proof. Fix a metric $g^{M}$ and so an induced $g^{Q}$ such that $Q \rightarrow M$ is a Riemannian submersion. Then $q: Q \times X \rightarrow X_{Q}$ is a Riemannian submersion with horizontal distribution $V \oplus T X$. It is also a principal $G$-bundle, and that distribution is a connection. Then the Levi-Civita covariant derivatives satisfy $\nabla^{X_{Q}}=q_{*} \Pi_{V \oplus T X}^{T Q \oplus T X} \nabla^{Q \times X}$, as follows by checking that the right hand side preserves the metric and is torsionfree. Hence the relative Levi-Civita covariant derivative on $X_{Q} \rightarrow M$ is

$$
\begin{aligned}
\nabla^{X_{Q} / M} & =\Pi_{T\left(X_{Q} / M\right)}^{T X_{Q}} q_{*} \Pi_{V \oplus T X}^{T Q \oplus T X} \nabla^{Q \times X} \\
& =q_{*} \Pi_{T X}^{V \oplus T X} \Pi_{V \oplus T X}^{T Q \oplus T X} \nabla^{Q \times X} \\
& =q_{*} \Pi_{T X}^{T Q \oplus T X} \nabla^{Q \times X} \\
& =q_{*} \nabla=\nabla_{Q} .
\end{aligned}
$$

For (ii) consider the iterated fiber bundle $Q \times X \stackrel{q}{\rightarrow} X_{Q} \rightarrow S_{Q} \rightarrow M$. Then using (11), (17), and the transitivity of compression, we have

$$
\nabla_{Q}^{X / S}=q_{*} \nabla^{X / S}=q_{*} \Pi_{T(X / S)}^{T X} \nabla=\Pi_{T\left(X_{Q} / S_{Q}\right)}^{T\left(X_{Q} / M\right)} q_{*} \nabla=\Pi_{T\left(X_{Q} / S_{Q}\right)}^{T\left(X_{Q} / M\right)} \nabla^{X_{Q} / M}=\nabla^{X_{Q} / S_{Q}}
$$

Proof of Theorem 14. The differential Borel quotient $\left(X_{G}\right)_{\nabla} \rightarrow\left(S_{G}\right)_{\nabla}$ inherits the relative Riemannian metric and, by our basic construction, a horizontal distribution. The relative Levi-Civita covariant derivative, determined by its pullbacks $\nabla_{Q}^{X / S}$ induced by maps $M \rightarrow B_{\nabla} G$, is $\nabla_{G}^{X / S}$; this is Lemma 16(ii). Therefore, its curvature is $\Omega_{G}^{X / S}$. Now (15) follows by testing against maps $M \rightarrow B_{\nabla} G$ and applying (12) to the pullback fiber bundles of smooth manifolds.

Remark 19. The "covariant anomaly formula" (e.g. [BZ, (3.60)], [AgG, (3.57)]) in quantum field theory is the moment map $\mu$. In the cited formulas the base $S$ is the space of covariant derivatives on 
a fixed vector bundle $E \rightarrow Y$, and $X=S \times Y$. The group $G$ is the infinite dimensional group of gauge transformations and the anomaly is the obstruction to descending Det $\rightarrow S$ to the quotient by $G$, i.e., the moment map precisely $\mu$. Theorem 14 applies by restricting to finite dimensional subgroups of $G$. Fix a connection in $S$ with curvature $F$, and fix an infinitesimal gauge transformation $v$. The equivariant curvature (9) evaluated on $v$ is $F-v$, and in the formula (15) we use the Chern character in place of the $\hat{A}$ polynomial. Assuming $\operatorname{dim} X=2 n$ we find $\mu=-2 \pi i\left(\frac{i}{2 \pi}\right)^{n} \frac{1}{n !} \int_{X} \operatorname{tr} v F^{n}$, which agrees with $[\mathrm{AgG},(3.57)]$ (up to $2 \pi i$ ). There is a similar formula for the "gravitational" case, in which $S$ is the space of Riemannian metrics on a fixed manifold $Y$ and $G$ is the group of diffeomorphisms ([BZ, (5.19)], [AgG, (5.32)]). Mathematical treatments of anomalies often first descend to the quotient $S / G$, essentially using the construction (11), in which case the anomaly is the determinant line bundle with covariant derivative associated to the family of Dirac operators over that quotient. The precise relationship between the moment map for the $G$-action on the determinant bundle over $S$ and the determinant bundle over $S / G$ ties together different approaches to anomalies.

\section{References}

[AgG] Luis Alvarez-Gaume and Paul Ginsparg, The structure of gauge and gravitational anomalies, Annals of Physics 161 (1985), no. 2, 423-490.

[AgW] L. Alvarez-Gaumé and E. Witten, Gravitational anomalies, Nucl. Phys. B234 (1983), 269.

[ASZ] Orlando Alvarez, IM Singer, and Bruno Zumino, Gravitational anomalies and the family's index theorem, Communications in Mathematical Physics 96 (1984), no. 3, 409-417.

[BF] Jean-Michel Bismut and Daniel S. Freed, The analysis of elliptic families. I. Metrics and connections on determinant bundles, Comm. Math. Phys. 106 (1986), no. 1, 159-176.

[BT] Raoul Bott and Loring W. Tu, Equivariant characteristic classes in the Cartan model, Geometry, analysis and applications (Varanasi, 2000), World Sci. Publ., River Edge, NJ, 2001, pp. 3-20. arXiv:math/0102001.

[BV] Nicole Berline et Michèle Vergne, Zeros d'un champ de vecteurs et classes characteristiques equivariantes, Duke Math. Journal 50 (1983), 539-549.

[BZ] William A Bardeen and Bruno Zumino, Consistent and covariant anomalies in gauge and gravitational theories, Nuclear Physics B 244 (1984), no. 2, 421-453.

[C1] Henri Cartan, Notions d'algèbre différentielle; application aux groupes de Lie et aux variétés où opère un groupe de Lie, Colloque de topologie (espaces fibrés), Bruxelles, 1950, Georges Thone, Liège, 1951, pp. 15-27. Reprinted in [GS].

[C2] La transgression dans un groupe de Lie et dans un espace fibré principal, Colloque de topologie (espaces fibrés), Bruxelles, 1950, Georges Thone, Liège, 1951, pp. 57-71. Reprinted in [GS].

[FH] Daniel S. Freed and Michael J. Hopkins, Chern-Weil forms and abstract homotopy theory, Bull. Amer. Math. Soc. (N.S.) 50 (2013), no. 3, 431-468, arXiv:1301.5959.

[GS] Victor W. Guillemin and Shlomo Sternberg, Supersymmetry and equivariant de Rham theory, Mathematics Past and Present, Springer-Verlag, Berlin, 1999. With an appendix containing two reprints by Henri Cartan [ MR0042426 (13,107e); MR0042427 (13,107f)].

[MQ] Varghese Mathai and Daniel Quillen, Superconnections, Thom classes, and equivariant differential forms, Topology 25 (1986), no. 1, 85-110.

[Q] D. Quillen, Determinants of Cauchy-Riemann operators on Riemann surfaces, Funktsional. Anal. i Prilozhen. 19 (1985), no. 1, 37-41, 96.

Department of Mathematics, University of Texas, Austin, TX 78712

E-mail address: dafr@math.utexas.edu 